\title{
Efecto de tratamientos pregerminativos de hidratación-deshidratación sobre la viabilidad y germinación de semillas de Setaria sphacelata
}

Tamborelli, M. R. y Fiant, S. E.

\begin{abstract}
RESUMEN
Setaria sphacelata es una gramínea forrajera megatérmica cultivada en diversas regiones dada su plasticidad. Al igual que otras forrajeras perennes estivales, tiene lenta implantación. Los tratamientos pregerminativos de hidratacióndeshidratación (HD) mejoran la germinación y el establecimiento de muchas especies. El objetivo de este trabajo fue evaluar el efecto de HD con agua y nitrato de potasio en semillas de Setaria sphacelata. Se aplicó un diseño factorial de tratamientos $2 \times 3 \times 2$. Un primer factor fue el agente imbibitorio: agua (A) y nitrato de potasio $2 \%$ (N); el segundo, el tiempo de imbibición: 24, 48 y 72 horas; y el tercero, los lotes evaluados (dos). Se sembraron cuatro repeticiones de 50 semillas por tratamiento y un testigo (T). Se registró energía germinativa, porcentaje de germinación, semillas frescas y muertas (SM). Entre lotes sólo se encontraron diferencias significativas en \% SM. Los tratamientos A24, A48, A72 y N24 superaron al T en porcentaje y velocidad de germinación, mientras que los N48 y N72 fueron similares o inferiores. El mejor tratamiento resultó ser HD con agua por 72 horas. La inmersión por más de 24 horas en nitrato de potasio 2 \% perjudicó la viabilidad de las semillas.
\end{abstract}

Palabras claves: forrajera megatérmica; priming; germinación; dormición; nitrato de potasio.

Tamborelli, M. R. and Fiant, S. E., 2020. Effect of hydration-dehydration pregerminative treatments on the viability and germination of Setaria sphacelata seeds. Agriscientia 37 (2): 35-43

\section{SUMMARY}

Setaria sphacelata is a tropical forage grass, cultivated in diverse regions due to its plasticity. Like other summer perennial forages, it has a slow establishment. Hydration-dehydration (HD) pregerminative treatments improve the germination and establishment of many species. The objective of this work was to evaluate the effect of HD with water and potassium nitrate on Setaria sphacelata seeds. A factorial treatment design of $2 \times 3 \times 2$ was applied. The first 
factor was the imbibitory agent: water $(A)$ and potassium nitrate at $2 \%(N)$; the second the imbibition time: 24,48 and 72 hours; and the third the lot evaluated (two). Four repetitions of 50 seeds per treatment and one control ( $T$ ) were sown. Germinative energy, germination percentage, fresh and dead seeds (SM) were recorded. Between lots, only significant differences were found in SM. The treatments A24, A48, A72 and N24 were better than T in speed and percentage of germination, while N48 and N72 were similar or inferior. It is concluded that HD treatment with water for 72 hours proved to be the best treatment. Immersion for more than 24 hours in potassium nitrate at $2 \%$ impaired seed viability.

Key words: tropical forage; priming; germination; dormancy; potassium nitrate.

Tamborelli, M. R. (ORCID: 0000-0003-3876-6653): Estación Experimental Agropecuaria Mercedes, Instituto Nacional de Tecnología Agropecuaria (INTA). Juan Pujol al Este s/n (3470), Mercedes, Corrientes, Argentina. Fiant, S. E. (ORCID: 0000-0002-3127-7315): Facultad de Ciencias Agropecuarias, Universidad Nacional de Córdoba. Ing. Agr. Félix Aldo Marrone 746, Ciudad Universitaria, (5001) Córdoba, Argentina; Bolsa y Cámara de Cereales de Córdoba, Francisco Ortíz de Campo 317, (5000) Córdoba, Argentina. Correspondencia a: tamborelli.melina@inta.gob.ar

\section{INTRODUCCIÓN}

Las pasturas megatérmicas, junto con los pastizales naturales conforman la cadena forrajera para la producción ganadera en la región extra pampeana argentina (Fiant, Núñez, Pereyra, Bruno y Tablada, 2018). Alrededor del $34 \%$ de la ganadería argentina se lleva a cabo en las regiones subtropicales del país (NEA y NOA), y de éstas, las subtropicales subhúmedas y semiáridas son las que presentan a futuro mayores posibilidades de incremento de la productividad mediante el uso de pasturas megatérmicas introducidas. El aumento del stock ganadero de esta región se ha correspondido, en gran parte, con un aumento en la implantación de pasturas tropicales (Arroquy e Imaz, 2015). La marcada distribución de la producción de los pastizales naturales a través del año y su baja calidad, sobre todo durante el período invernal, hace que las pasturas implantadas jueguen un rol muy importante en estas regiones. En áreas donde los pastos naturales son de baja calidad, el impacto del reemplazo de éstos por pasturas subtropicales es muy alto (Pizzio, Borrajo y Barbera, 2006).

Setaria sphacelata (Schumach.) Stapf \& C. E. Hubb es una gramínea forrajera megatérmica nativa de África tropical y subtropical, ampliamente utilizada en pastoreo de ganado de carne, como así también para la conservación del forraje como heno (Hacker y Jones, 1969). Muestra una gran adaptación a diversos tipos de suelo, desde francos a arcillosos, creciendo bien en suelos arenosos profundos o superficiales, en suelos ácidos sueltos o pesados, y tolera bajos niveles de fertilidad, especialmente fosfórica. Debido a su gran plasticidad es cultivada con éxito en una amplia diversidad de regiones agroecológicas. Según el ambiente y con un manejo adecuado, se logran pasturas con más de diez años de persistencia (Borrajo y Pizzio, 2006). Es perenne pero de alta producción estival; su crecimiento comienza en primavera y su producción anual de materia seca fluctúa entre 6000 y $10000 \mathrm{~kg} \mathrm{ha}^{-1}$.

Uno de los objetivos principales en la implantación de gramíneas forrajeras es lograr que la germinación y la emergencia de las plántulas se resuelvan rápida y uniformemente. Variaciones tanto en la velocidad de germinación como en la de formación de hojas y macollos pueden determinar capacidades diferenciales para competir durante el establecimiento, alcanzar una rápida cobertura y permitir un aprovechamiento de forraje anticipado (Horst y Dunning, 1989).

La correcta implantación de pasturas es una de las tareas más difíciles y delicadas si se compara con la siembra de cualquier otro 
cultivo agrícola (Pozzolo, 2006). Las forrajeras perennes megatérmicas tienen normalmente bajos coeficientes de logro, es decir escasa cantidad de plantas logradas en relación a las semillas viables sembradas. Son lentas en implantarse, lo que las hace vulnerables a déficit y excesos hídricos, situación que se agrava por lo variable de las condiciones ambientales primaverales, los suelos pobres, sistemas de siembra utilizados y la elevada presión de malezas reinante en los ambientes productivos de estas pasturas. Se agrega el problema primario de baja germinación, que es frecuente (Barbera, 2015).

Una alternativa para mejorar el comportamiento germinativo de muchas especies de interés agrícola son los tratamientos pregerminativos de hidratación-deshidratación de las semillas, que han probado ser eficientes para revigorizar semillas envejecidas, acelerar e incrementar la germinación y los rendimientos de las plantas, tanto bajo condiciones ecológicas óptimas como adversas (Chatterjee y Singh, 1983; Sánchez, Orta y Muñoz, 2001). Dichas técnicas son conocidas como acondicionamiento de semillas o seed priming. El acondicionamiento fisiológico implica la absorción de agua por la semilla, bajo condiciones controladas, para incentivar el metabolismo de las semillas durante las fases I y || de imbibición (Bewley y Black, 1994) pero impidiendo que ocurra la protrusión de la raíz primaria. De esa manera se activa la digestión de reservas, su traslocación y asimilación, para que las semillas que componen el lote alcancen un estado metabólico relativamente uniforme cuando el acceso de agua es voluntariamente interrumpido.

Las metodologías empleadas en el acondicionamiento fisiológico pueden diferir entre sí. Las variaciones consisten en las formas de suministro del agua que pueden incluir equilibrio higroscópico con la atmósfera o imbibición en sustrato o inmersión en agua pura y/o en soluciones salinas u osmóticas, denominándose osmoacondicionamiento al tratamiento que emplea este último tipo de soluciones en la imbibición. Otras variaciones pueden estar en el tipo de secado posterior a la hidratación, en el número de ciclos de hidratación y deshidratación, en la duración del período de hidratación y en el estadio de la germinación alcanzado durante el acondicionamiento (Guimarães, 2000). Cuando las condiciones del tratamiento son favorables, el proceso de movilización de reservas, la activación y síntesis de novo de algunas enzimas y la síntesis de ADN y ARN son iniciadas durante el acondicionamiento. Cuando el obstáculo a la absorción de agua es removido ocurre un rápido crecimiento del embrión (Khan, 1992).

En la práctica se aplican diferentes sustancias y métodos para que las semillas logren el mismo nivel de humedad y estado fisiológico. Los mejores resultados experimentales, generalmente, se alcanzan con soluciones poliméricas, como el polietilenglicol (PEG) (Heydecker, 1974; Bradford, 1986), pero en condiciones agronómicas, resultan más simples y baratos los métodos de imbibición parcial en agua ya que han demostrado ser eficientes para incrementar, acelerar y uniformar la germinación (Gray, Rowse y Drew, 1990; Jett, Welbaum y Morse, 1996; Sánchez, Calvo, Orta y Muñoz, 1997; Orta, Sánchez, Muñoz y Calvo, 1998). Algunos autores defienden la utilización de sales, considerándolas más ventajosas en relación al PEG, porque no reducen la disponibilidad de oxígeno, además de resaltar los beneficios del nitrato de potasio $\left(\mathrm{KNO}_{3}\right)$ en la germinación de algunas especies, especialmente cuando hay dormición (Marcos Filho, 2015), como es el caso de Setaria sphacelata, especie en la cual se desconoce el impacto que podrían producir los tratamientos de HD en la germinación de las semillas.

La dormición característica de pasturas de verano (Petruzzi, Stritzler, Adema, Ferri y Pagella, 2003), es una estraregia que tienen algunas plantas para prevenir la germinación durante períodos en los que las condiciones del ambiente no son favorables, incluyendo la etapa de desarrollo de plántulas. Marcos Filho (2015) cita a las gramíneas forrajeras como ejemplo para el caso de semillas que se desprenden de la planta madre con su estructura morfológica completa, pero con un embrión fisiológicamente inmaduro que presenta dormición. Esta inmadurez fisiológica ha sido atribuida a la desuniformidad de maduración de semillas de la misma planta, lo que ocasiona la cosecha de una parte de ellas con maduración incompleta. Éstas pueden presentar, por ejemplo, un desequilibrio entre sustancias promotoras e inhibidoras de la germinación como lo son el ácido abscísico y las giberelinas. Teniendo en cuenta estos aspectos se podría asumir que este tipo de dormición se encontraría, según la clasificación propuesta por Baskin y Baskin (2004), dentro del tipo endógena fisiológica, pero se desconoce hasta el momento el tipo de dormición que gobierna el pulso de germinación en Setaria sphacelata.

El objetivo del presente estudio fue evaluar el efecto de tratamientos pregerminativos de hidratación-deshidratación con agua y nitrato de potasio sobre la viabilidad y germinación de semillas de Setaria sphacelata pertenecientes a dos lotes de distintos años de cosecha. 


\section{MATERIALES Y MÉTODOS}

El ensayo se llevó a cabo en el Laboratorio de Análisis de Semillas de la EEA INTA Mercedes,

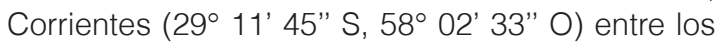
meses de enero y febrero de 2017.

Se utilizaron semillas de Setaria sphacelata, sin tratamiento previo, pertenecientes a dos lotes distintos, cosechados en enero de 2015 y 2016, con 24 y 12 meses de almacenamiento respectivamente. Previo al ensayo, las semillas estuvieron almacenadas en un depósito con ambiente controlado $\left(18-20^{\circ} \mathrm{C}\right.$ y $60-70 \%$ de HR). Al momento de inicio del ensayo las semillas se encontraban con $11 \%$ de humedad, la cual fue determinada por el método de estufa según Reglas ISTA (International Rules for Seed Testing, 2017).

El diseño experimental fue completamente aleatorizado con una estructura factorial de tratamientos $2 \times 3 \times 2$. El primer factor corresponde al agente imbibitorio (agua destilada y solución de nitrato de potasio al $2 \%$ ); el segundo factor corresponde al tiempo de imbibición (24, 48 y 72 horas) y el tercero, a los dos lotes evaluados. Para cada tratamiento se utilizaron 5 gramos de semillas (aproximadamente cinco mil semillas), las cuales se pusieron a embeber con $50 \mathrm{ml}$ del agente imbibitorio correspondiente en cajitas plásticas con tapa de $8 \times 10 \times 4 \mathrm{~cm}$ de ancho, largo y altura respectivamente. Las semillas se pusieron a embeber en un ambiente con temperatura constante de $25^{\circ} \mathrm{C}$ y un régimen de luz/oscuridad de $8 / 16$ horas respectivamente.

Finalizado el período de imbibición las semillas tratadas se secaron superficialmente y se pesaron para conocer el porcentaje de incremento de peso post tratamiento. Luego se colocaron sobre toallas de papel absorbente en un ambiente controlado $\left(18-20{ }^{\circ} \mathrm{C}\right.$ y $60-70 \%$ de HR) durante 72 horas; transcurrido este período se pesó nuevamente cada muestra para constatar que presentaban el peso inicial previo acondicionamiento.

A continuación, 24 horas después del secado, se pusieron a germinar cuatro repeticiones de 50 semillas por cada tratamiento en cámara de germinación con alternancia de $20-35{ }^{\circ} \mathrm{C}$ y un período de luz/oscuridad de 8/16 horas, como indican las Reglas ISTA (2017). También se pusieron a germinar a modo de testigo, 4 repeticiones de 50 semillas sin tratamiento pregerminativo.

Fueron evaluadas las siguientes variables:

- Primer recuento de germinación o energía germinativa (\%EG): calculado como el número de plántulas normales germinadas a los 7 días, expresado en porcentaje.
- Poder germinativo (\%PG): calculado como el número de plántulas normales germinadas a los 21 días, expresado en porcentaje.

- Semillas frescas (\%SF): determinadas como la diferencia entre el número de semillas viables por test de Tetrazolio y el número de plántulas normales germinadas, expresadas en porcentaje.

- Semillas muertas (\%SM): determinadas a través de un ensayo de viabilidad por test de Tetrazolio según Reglas ISTA (2017), expresadas en porcentaje (100 - semillas viables).

\section{Diseño experimental y análisis estadístico}

Para comparar el resultado de los tratamientos se realizó un análisis de la varianza (ANAVA) para un diseño completamente aleatorizado y comparación de medias mediante la prueba DGC con un nivel de significancia de $\alpha=0,05$.

Previo al análisis de los datos y una vez calculados los residuos se verificó el cumplimiento de los supuestos de normalidad, independencia y homogeneidad de varianzas de los errores, mediante la prueba de hipótesis de ShapiroWilks e interpretaciones gráficas de la dispersión de residuos y predichos. Todos los análisis se realizaron utilizando el software InfoStat (Di Rienzo et al., 2017).

\section{RESULTADOS Y DISCUSIÓN}

En las Tablas 1 y 2 se pueden observar el porcentaje de incremento en peso base húmedo promedio de las semillas del lote 2016 y 2015 respectivamente, después de la aplicación de los distintos tratamientos de priming. En dichas tablas también se puede corroborar cómo a las 72 horas post tratamiento las semillas volvieron a un valor muy próximo a su peso inicial. Si se observa el promedio de los distintos tratamientos se encontró que el lote 2015 tuvo un mayor porcentaje de incremento (40,83\%) que el lote 2016 (33,93\%). Si bien la capacidad de hidratación de las semillas recién cosechadas no fue objeto de este estudio, la necesidad de calibrar la metodología para los tratamientos pregerminativos de las semillas almacenadas Ilevó a caracterizar los incrementos de peso logrados con cada tratamiento. En tal sentido, la variabilidad hallada entre lotes podría atribuirse a que las semillas se formaron en planta madre bajo diferentes condiciones ambientales, dadas tanto por los años de cosecha como por los diferentes momentos de formación de semillas 
dentro de una misma temporada, recordando el escalonamiento en la floración que presenta esta especie y las forrajeras megatérmicas en general. Otra posible causa de las diferencias en el incremento de peso de las semillas hidratadas podría ser una limitación física a la entrada de agua, determinada por la diferente conformación de la pared celular, constituida por laminilla media, pared primaria y, en algunos casos, pared secundaria. Estas paredes pueden tener variaciones en el espesor y en los contenidos de celulosa, hemicelulosa, lignina y sustancias pécticas, características que se relacionan con la dureza y resistencia mecánica (Salisbury y Ross, 1992; Taiz y Zeiger, 1998). Si bien no se encontraron referencias al respecto en Setaria sphacelata, en investigaciones realizadas por Galussi, Arguello, Cerana, Maximino y Moya (2015) sobre semillas de alfalfa, de un mismo cultivar, pero de distintos años de cosecha, se pusieron en evidencia cambios en el grosor de la testa en función del nivel de permeabilidad al agua (clasificada en rápida, lenta y muy lenta hidratación). Las características anatómicas del tegumento y las proporciones de los componentes hallados determinaron los niveles de permeabilidad del tegumento. Por otra parte, si bien el lote 2015 tuvo un mayor incremento de peso, en los dos lotes se constató el retorno al peso inicial luego de las 72 horas de imbibición. En los tratamientos de priming la imbibición se da mediante la inmersión de las semillas en soluciones osmóticas o en cantidades determinadas de agua durante cierto tiempo permitiendo que un mayor número de semillas alcance rápidamente el mismo nivel de humedad y active el aparato metabólico relacionado con el proceso pregerminativo (Burgas y Powell, 1984). De este modo, es factible mejorar la calidad fisiológica de la semilla al alcanzar una mayor uniformidad en el porcentaje de germinación (Sánchez, Mejía, Hernández, Peña y Carballo, 2007). Por su parte Brocklehurst y Dearman (1984) observaron que el acondicionamiento puede ser más favorable sobre el desempeño de semillas de cebolla de bajo potencial fisiológico, cuando es comparado con semillas más vigorosas.

En cuanto a las variables energía germinativa y poder germinativo se observó diferencia significativa entre tratamientos $(<0,0001)$, no así entre lotes. Como se puede observar en las Figuras 1 y 2 para ambos lotes, los tratamientos que más incrementaron el porcentaje de germinación fueron todos los que implicaban imbibición en agua (A24, A48, A72) y el tratamiento con nitrato de potasio por 24 horas (N24) que superaron en hasta 20 puntos al testigo, pero sin diferencia significativa entre ellos. De modo similar vemos en la Figura 3 que los tratamientos A24, A48, A72 y N24 superaron al testigo en porcentaje de energía germinativa, mientras que los N48 y N72 estuvieron por debajo del mismo, demostrando particularmente que el priming con agua (o hidropriming) ofrecería la ventaja de una rápida germinación, aspecto deseable al momento de implantación de pasturas y del que no se encontraron antecedentes en la bibliografía para Setaria sphacelata. Los resultados obtenidos en este trabajo en tratamientos de imbibición con agua, coinciden con las afirmaciones de Caseiro (2003) quien señala que en numerosos estudios la utilización de agua en el acondicionamiento fisiológico ha resultado una interesante opción, favoreciendo el desempeño de las semillas sin la interferencia de productos químicos empleados en el osmoacondicionamiento. En idéntico sentido, Medeiros Filho, Carvalho, Teófilo y Rossetti (2000) observaron que el acondicionamiento de semillas de sorgo en agua destilada fue el más eficiente en promover el aumento de vigor de éstas en relación al osmoacondicionamiento. En semillas de tomate, la velocidad y uniformidad de germinación fueron más favorecidas por la imbibición de las semillas en agua destilada que en soluciones osmóticas (Coolbear, Newell y Bryant, 1987). De forma similar Trigo, Nedel, Garcia y Trigo (1999) evidenciaron que el acondicionamiento de semillas de cebolla en agua proporcionó incrementos en el porcentaje de germinación, la velocidad de germinación, en el test de frío y en la longitud de la raíz primaria. Mgaya, Thobunluepop, Sreewongchai, Sarobol y Onwimol (2016) evaluaron en semillas de arroz tratamientos de acondicionamiento en agua por 24 horas y en nitrato de potasio al $2 \%$ por 48 horas, para las variables porcentaje y velocidad de germinación todos los tratamientos superaron al testigo, pero el mejor fue el acondicionamiento en agua. Para semillas de coliflor, Fujikura, Karssen, Kraak y Basra (1993) verificaron que el hidropriming incrementó la velocidad de germinación. Estos autores destacaron que además de ser un método simple y barato no requiere reactivos 0 equipamientos sofisticados.

Ahora bien, los resultados del presente trabajo demuestran que los tratamientos que implicaron mayor tiempo de imbibición con nitrato de potasio (N48 y N72) no presentaron diferencias significativas con el testigo o resultaron inferiores tanto para el porcentaje de germinación (Figuras 1 y 2) como para el porcentaje de energía germinativa (Figura 3). A su vez, estos tratamientos afectaron la viabilidad de las semillas, lo cual se evidencia en el elevado porcentaje de semillas muertas 
registradas (Figura 1 y 2). Para ésta última variable se observaron diferencias significativas entre tratamientos $(P<0,0001)$ y entre lotes $(P<0,0001)$ presentando mayores valores el lote con 24 meses de almacenamiento (Figura 4). Esto es esperable por tratarse de semillas expuestas durante más tiempo al proceso natural de envejecimiento que las conducen finalmente a su muerte (Olivera, 2011). Existen evidencias que indican que las sales también pueden provocar efectos negativos, por ejemplo, soluciones salinas fueron tóxicas para semillas de Sorghum bicolor (Haigh y Barlow, 1987), lo que condice con los resultados de los tratamientos con nitrato de potasio N48 y N72. Asimismo, Brocklehurst y Dearman (1984) verificaron que el acondicionamiento osmótico con fosfato mono potásico redujo el porcentaje de germinación y emergencia de plántulas en cebolla, apio y zanahoria. En el mismo sentido, Nirmala y Umarani (2008) evaluaron en semillas de okra (Abelmoschus esculentus) y remolacha (Beta vulgaris) tratamientos con agua y nitrato de potasio y observaron que el nitrato de potasio fue perjudicial para las semillas de okra independientemente de la concentración y duración del tratamiento, en tanto que para remolacha el mejor tratamiento fue con agua durante 12 horas. Según Thanos y Georghiou (1988) la promoción de germinación por las sales se debe a sus propiedades osmóticas y no a sus propiedades químicas, aunque estas últimas pueden afectar las estructuras celulares de las semillas y, por consiguiente, la viabilidad de las mismas (Khan, Peck, Taylor y Samimy, 1983; Brocklehurst y Dearman, 1984 y Bradford, 1995).

En el presente estudio, al analizar el porcentaje de semillas frescas se observaron diferencias significativas entre tratamientos $(<0,0001)$ pero no entre lotes $(0,2238)$, como se esperaba el mayor porcentaje lo obtuvo el testigo (27 \%), a continuación se ubicaron los N24 y A72 con 17 y $15 \%$ respectivamente. Finalmente se destacaron con una fuerte disminución en el porcentaje de semillas frescas, los tratamientos A24, A48, N48 y N72. De éstos últimos hay que resaltar que en el caso de los tratamientos N48 y N72, esta disminución fue acompañada de un efecto

Tabla 1. Peso inicial (g), peso post tratamiento (g), incremento de peso (\%) y peso final (g) después de 72 horas de finalizado el priming en semillas de Setaria sphacelata cosecha 2016, sometidas a diferentes tratamientos de priming, media y desvío estándar (D. E.) de todos los tratamientos

\begin{tabular}{|c|c|c|c|c|c|}
\hline Agente imbibitorio & $\begin{array}{c}\text { Período de } \\
\text { imbibición (horas) }\end{array}$ & $\begin{array}{c}\text { Peso } \\
\text { inicial (g) }\end{array}$ & $\begin{array}{c}\text { Peso post } \\
\text { tratamiento }(\mathrm{g})\end{array}$ & $\begin{array}{c}\text { Incremento de } \\
\text { peso (\%) }\end{array}$ & Peso final (g) \\
\hline \multirow[t]{3}{*}{ Agua } & 72 & 5 & 6,65 & 33,0 & 4,91 \\
\hline & 48 & 5 & 6,76 & 35,2 & 4,93 \\
\hline & 24 & 5 & 6,67 & 33,4 & 4,94 \\
\hline \multirow[t]{3}{*}{$\mathrm{KNO}_{3}$} & 72 & 5 & 6,68 & 33,6 & 4,96 \\
\hline & 48 & 5 & 6,84 & 36,8 & 4,99 \\
\hline & 24 & 5 & 6,58 & 31,6 & 5,0 \\
\hline Media & & & 6,70 & 33,9 & 4,96 \\
\hline D. E. & & & 0,09 & 1,82 & 0,04 \\
\hline
\end{tabular}

Tabla 2. Peso inicial (g), peso post tratamiento (g), incremento de peso (\%) y peso final (g) después de 72 horas de finalizado el priming en semillas de Setaria sphacelata cosecha 2015, sometidas a diferentes tratamientos de priming, media y desvío estándar (D. E.) de todos los tratamientos

\begin{tabular}{lccccc}
\hline $\begin{array}{l}\text { Agente } \\
\text { imbibitorio }\end{array}$ & $\begin{array}{c}\text { Período de imbibición } \\
\text { (horas) }\end{array}$ & $\begin{array}{c}\text { Peso inicial } \\
\text { (g) }\end{array}$ & $\begin{array}{c}\text { Peso post } \\
\text { tratamiento (g) }\end{array}$ & $\begin{array}{c}\text { Incremento de } \\
\text { peso (\%) }\end{array}$ & Peso final (g) \\
\hline \multirow{3}{*}{ Agua } & 72 & 5 & 7,06 & 41,2 & 4,91 \\
& 48 & 5 & 7,06 & 41,0 & 4,93 \\
& 24 & 5 & 6,89 & 37,8 & 4,94 \\
KNO $_{3}$ & 72 & 5 & 7,44 & 48,8 & 4,96 \\
& 48 & 5 & 6,93 & 38,6 & 4,99 \\
Media & 24 & 5 & 6,88 & 37,6 & 5 \\
D. E. & - & - & 7,04 & 40,83 & 4,96 \\
\end{tabular}


perjudicial de la aplicación de los tratamientos con un fuerte aumento en el porcentaje de semillas muertas. Sin embargo, en los tratamientos A24 y A48 la disminución del porcentaje de semillas frescas podría atribuirse a una disminución en la dormición, lo que se ve reflejado en el aumento del porcentaje de germinación (Figuras 1 y 2). Marcos Filho (2015) describe que uno de los efectos

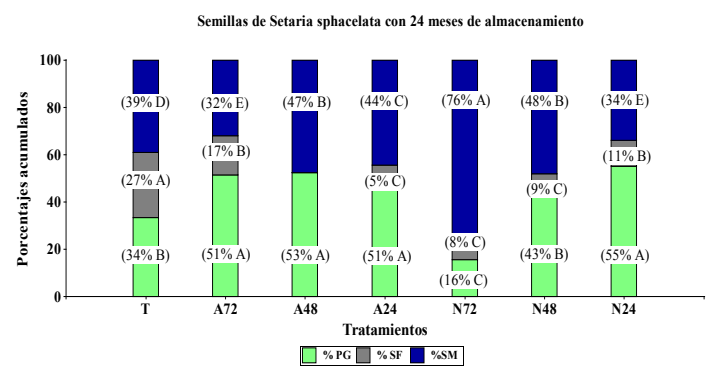

Figura 1. Porcentajes de germinación (\%PG), semillas frescas (\%SF) y semillas muertas (\%SM) observados en semillas de Setaria sphacelata pertenecientes a un lote con 24 meses de almacenamiento luego de aplicar los tratamientos: inmersión en agua 72 horas (A72), 48 horas (A48) y 24 horas (A24); inmersión en nitrato de potasio al $2 \% 72$ horas (N72), 48 horas (N48), 24 horas (N24) y un testigo sin tratar $(\mathrm{T})$. Letras diferentes indican diferencia significativa entre tratamientos $(p<0,05)$

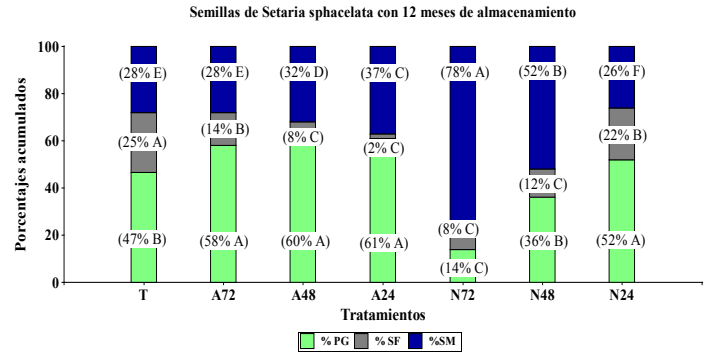

Figura 2. Porcentajes de germinación (\%PG), semillas frescas (\%SF) y semillas muertas (\%SM) observados en semillas de Setaria sphacelata pertenecientes a un lote con 12 meses de almacenamiento luego de aplicar los tratamientos: inmersión en agua 72 horas (A72), 48 horas (A48) y 24 horas (A24); inmersión en nitrato de potasio al $2 \% 72$ horas (N72), 48 horas (N48), 24 horas (N24) y un testigo sin tratar (T). Letras diferentes indican diferencia significativa entre tratamientos $(p<0,05)$

\section{CONCLUSIONES}

Setaria sphacelata (Schumach.) Stapf \& C. E. Hubb es una especie forrajera que responde satisfactoriamente a tratamientos pregerminativos de hidratación y deshidratación con agua. Independientemente de la edad fisiológica del lote de semillas, la imbibición con agua demuestra resultados de germinación y primer recuento o del acondicionamiento fisiológico es atenuar la profundidad de esa dormición. Como ejemplo de esto, Bradford (1990) trabajó con 42 lotes de semillas de pimiento de diferentes grados de maduración y lograron los mejores resultados, para incrementar y acelerar la germinación, cuando los tratamientos acondicionadores fueron aplicados en semillas frescas o inmaduras.

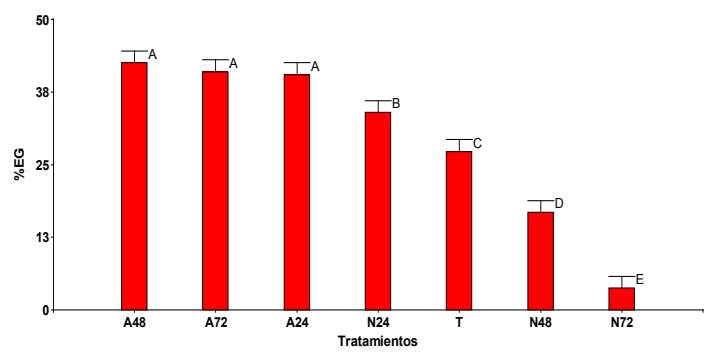

Figura 3. Porcentajes de energía germinativa (\%EG) observados en semillas de Setaria sphacelata, promedio de dos lotes cosecha 2015 y 2016 luego de aplicar los tratamientos: inmersión en agua 72 horas (A72), 48 horas (A48) y 24 horas (A24); inmersión en nitrato de potasio al $2 \% 72$ horas (N72), 48 horas (N48), 24 horas (N24) y un testigo sin tratar (T). Las columnas representan la media de los tratamientos $(n=8)$, y las barras verticales, el error estándar. Letras diferentes indican diferencia significativa entre tratamientos $(p<0,05)$

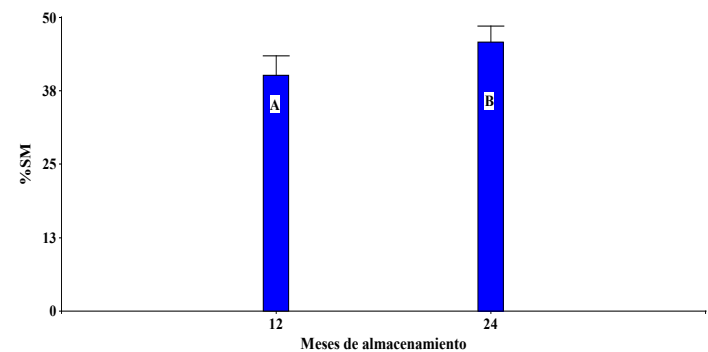

Figura 4. Porcentajes de semillas muertas (\%SM) observados en dos lotes de semillas de Setaria sphacelata con 12 y 24 meses de almacenamiento. Las columnas representan la media $(n=28)$ de todos los tratamientos, y las barras verticales, el error estándar. Letras diferentes indican diferencia significativa entre los lotes de distinto año de cosecha $(p<0,05)$

energía germinativa mayores a los observados con el uso de nitrato de potasio como agente de imbibición.

El mejor tratamiento de hidratación y deshidratación resultó ser el uso de agua durante 72 horas de imbibición. Esta combinación de agente y tiempo de imbibición produce menores niveles de semillas muertas, lo que en conjunto con los mayores niveles de germinación sugiere el alto 
potencial agronómico de esta técnica que permite homogenizar y acelerar la germinación de esta pastura. Su relativo bajo costo de producción y la sencillez de su aplicación hacen factible extender su aplicación a la práctica agrícola.

\section{AGRADECIMIENTOS}

Las autoras agradecen al Ing. Agr. Guillermo Mc Lean y al Ing. Zoot. Pablo Barbera por su colaboración en la revisión del manuscrito.

\section{BIBLIOGRAFÍA}

Arroquy, J. I. e Imaz, A. (2015). Valor nutritivo y aprovechamiento de pasturas tropicales. (Informe Técnico N. ${ }^{\circ} 60$ presentado en la II Jornada Nacional de Forrajeras Tropicales, 27-38). Rafaela, Santa Fe, Argentina: INTA Ediciones.

Barbera, P. (2015). Rol actual y potencial de las forrajeras tropicales en los sistemas ganaderos de ambientes húmedos (Informe Técnico N. ${ }^{\circ} 60$ presentado en la II Jornada Nacional de Forrajeras Tropicales, 39-52). Rafaela, Santa Fe, Argentina: INTA Ediciones.

Baskin, J. M. y Baskin, C. C. (2004). A classification system for seed dormancy. Seed Science Research, 14, 1-16. DOI: https://doi.org/10.1079/SSR2003150

Bewley, J. D. y Black, M. (1994). Seeds: physiology of development and germination. ( $3^{\mathrm{a}} \mathrm{ed}$.). Nueva York, EE.UU: PlenumPress.

Borrajo, C. y Pizzio, R. (2006). Manual de producción y utilización de Setaria. Proyecto ganadero de Corrientes, 1-11. Recuperado de: http://www. produccion-animal.com.ar/produccion_y_manejo_ pasturas/pasturas_cultivadas_megatermicas/178Manual_Setaria.pdf

Bradford, K. J. (1986). Manipulation of seed water relations via osmotic priming to improve germination under stress conditions. HortScience, 21, 1105-1112.

Bradford, K. J. (1990). A water relations analysis of seed germination rates. Plant Physiology, 94 (2), 840-849. DOI: https://doi.org/10.1104/pp.94.2.840

Bradford, K. J. (1995). Water relations in seed germination. En Kigel, J. y Galili, G. (Eds.), Seed development and germination, (351-396). Nueva York, EE. UU.: Marcel Dekker.

Brocklehurst, P. y Dearman, J. (1984). A comparison of different chemicals for osmotic treatment of vegetable seed. Annals of Applied Biology, 105 (2), 391-398. DOI: https://doi.org/10.1111/j.1744-7348.1984.tb03064.x

Burgas, R. y Powell, A. (1984). Evidence for repair processes in the invigoration of seeds by hidration.
Annals of Botany, 53 (5), 753-757. DOI: https://doi. org/10.1093/oxfordjournals.aob.a086741

Caseiro, R. F. (2003). Métodos para condicionamiento fisiológico de sementes de cebola e influencia da secagem e armazenamento. Tesis doctoral no publicada, Escuela Superior de Agricultura Luiz de Queiroz, Universidad de San Pablo, Piracicaba. DOI: https://doi.org/10.11606/T.11.2004.tde-18052004160932

Chatterjee, B. y Singh, A. (1983). Producción de cebada a partir de semillas tratadas antes de la siembra. The Journal of Agricultural Science, 100, 235-239.

Coolbear, P., Newell, A. y Bryant, J. (1987). An evaluation of the potential of low temperature pre sowing treatments of tomato seeds as a means of improving germination performance. Annals of Applied Biology, 110 (1), 185194. DOI: https://doi.org/10.1111/j.1744-7348.1987. tb03246.x

Di Rienzo, J. A., Casanoves, F., Balzarini, M. G., Gonzalez, L., Tablada, M. y Robledo, C. W. Infostat (versión 2017) [Software]. Córdoba, Argentina: Grupo InfoStat, FCA, Universidad Nacional de Córdoba. URL: http://www. infostat.com.ar

Fiant, S. E., Núñez, S. B., Pereyra, M. S., Bruno, C. I. y Tablada, E. M. (2018). Manual de buenas prácticas para la evaluación de calidad de semillas incrustadas de GattonPanic. Facultad de Ciencias Agropecuarias, Universidad Nacional de Córdoba, Argentina. Recuperado de: https://rdu.unc.edu.ar/ handle/11086/6791

Fujikura, Y., Kraak, H., Basra, A. y Karssen, C. (1993). Hydropriming, a simple and inexpensive priming method. Seed Science and Technology, 21 (3), 639642.

Galussi, A. A., Arguello, J. A., Cerana, M. M., Maximino, M. G. y Moya, M. E. (2015). Características anatómicas y químicas del tegumento seminal de Medicago sativa L. (alfalfa) Cv. Baralfa 85 y su asociación con la dormición. Phyton- International Journal of Experimentall Botany, 84, 163-175. Recuperado de: http://hdl.handle.net/11336/38236

Gray, D., Rowse, H. y Drew, R. (1990). A comparison of two large scale seed priming techniques. Annals of Applied Biology, 116 (3), 611-616. DOI: https://doi. org/10.1111/j.1744-7348.1990.tb06644.x

Guimarães, R. M. (2000). Tolerância à dessecação e co ndicionamentofisiológicoemsementes de cafeeiro (Coffeaarabica, L.). Tesis Doctoral, Universidad Federal de Lavras, Minas Gerais, Brasil. Recuperado de: http://www.sbicafe.ufv.br/handle/123456789/505\#

Hacker, J. B. y Jones, R. J. (1969). The Setaria sphacelata complex - a review. Tropical Grasslands, 3, 13-34.

Haigh, A. y Barlow, E. (1987). Germination and priming of tomato, carrot, onion, and sorghum seeds in a 
range of osmotica. Journal of the American Society for Horticultural Science, 112 (2), 202-208.

Heydecker, W. (1974). Germination of an idea: the priming of seeds. School of Agriculture Research, University of Nottingham, Nottingham, 1973, 50-67.

Horst, G. y Dunning, N. (1989). Germination and seedling growth of perennial ryegrasses in soluble salts. Journal of the American Society for Horticultural Science, 114, 338-342.

ISTA, International Rules for Seed Testing (2017). Bassersdorf, Switzerland: ISTA.

Jett, L. W., Welbaum, G. E. y Morse, R. D. (1996). Effects of matric and osmotic priming treatments on broccoli seed germination. Journal of the American Society for Horticultural Science, 121 (3), 423-429. DOI: https:// doi.org/10.21273/JASHS.121.3.423

Khan, A. A. (1992). Preplant physiological seed conditioning. Horticultural reviews, 13 (1), 131-181. DOI: https://doi.org/10.1002/9780470650509.ch4

Khan, A. A., Peck, N. H., Taylor, A. G. y Samimy, C. (1983). Osmoconditioning of Beet Seeds to Improve Emergence and Yield in Cold Soil. Agronomy Journal, 75(5), 788-794. DOI: https://doi.org/10.2134/agronj19 83.00021962007500050015x

Marcos Filho, J. (2015). Fisiologia de sementes de plantas cultivadas. Londrina, PR, Brasil: ABRATES

Medeiros Filho, S., Carvalho, L., Teófilo, E. y Rossetti, A. (2000). Efeito do osmocondicionamento no vigor de sementes de sorgo. Ciência Agronômica, 31 (1-2), 3342.

Mgaya, A., Thobunluepop, P., Sreewongchai, T., Sarobol, E. y Onwimol, D. (2016). Integral Effect of Seed Treatments and Production Systems for Sustainability of Rice Production under Acid Soil. Journal of Agronomy, 15 (3), 122-129. DOI: https://doi. org/10.3923/ja.2016.122.129

Nirmala, K. y Umarani, R. (2008). Evaluation of seed priming methods to improve seed vigour of okra (Abelmoschus esculentus) and beetroot (Beta vulgaris). Seed Science and Technology, 36, 56-65. DOI: https://doi.org/10.15258/sst.2008.36.1.06

Olivera, M. E. (2011) Efecto del incrustado sobre atributos fisiológicos de semillas de festuca alta (Festuca arundinacea Schreb). Tesis de maestría. Facultad de Ciencias Agopecuarias, Universidad Nacional de Córdoba, Argentina. Recuperado de: https:// agro.biblio.unc.edu.ar/cgi-bin/koha/opac-detail. pl?biblionumber $=10657$

Orta, R., Sánchez, J., Muñoz, B. y Calvo, E. (1998). Modelo de hidratación parcial en agua para tratamientos revigorizadores, acondicionadores y robustecedores de semillas. Acta Botánica Cubana, 121, 1-8.

Petruzzi H., Stritzler N., Adema E., Ferri C. y Pagella J. (2003). Mijo perenne - Panicumcoloratum. (Informe técnico N. ${ }^{\circ}$ 51). Anguil, La Pampa, Argentina: INTA Ediciones. Recuperado de: http://www.produccionanimal.com.ar/produccion_y_manejo_pasturas/ pasturas_cultivadas_megatermicas/87-publi51.pdf

Pizzio, R., Borrajo, C. I. y Barbera, P. (2006). Pasturas implantadas en Corrientes. Tierra Correntina, Año I, N ${ }^{\circ}$ 1, 19-21.

Pozzolo, O. R. (2006). Recomendaciones para la implantación de pasturas. (Informe técnico $N^{\circ} 6$, 143). Recuperado de: http://produccion-animal.com. ar/produccion_y_manejo_pasturas/pasturas $\% 20$ artificiales/42-implantacion_praderas.pdf

Salisbury, F. B. y Ross C. W. (2000). Fisiología de las plantas 1. Células: agua, soluciones y superficies. Madrid, España: Ediciones Paraninfo S.A.

Sánchez, J. A., Calvo, E., Orta, R. y Muñoz, B. (1997). Tratamientos pregerminativos de hidratacióndeshidratación para semillas de pepino (Cucucmis sativus L.). Acta Botánica Mexicana, 38, 13-20. DOI: https://doi.org/10.21829/abm38.1997.772

Sánchez, J. A., Orta, R. y Muñoz, B. C. (2001). Tratamientos pregerminativos de hidratación-deshidratación de las semillas y sus efectos en plantas de interés agrícola. Agronomía Costarricense, 25, 67-92. Recuperado de: https://www.redalyc.org/pdf/436/43625107.pdf

Sánchez, J., Mejía, A. J. A, Hernández, A., Peña, A. y Carballo, C. (2007). Acondicionamiento osmótico de semillas de tomate de cáscara. Agricultura Técnica en México, 33 (2), 115-123. Recuperado de: http:// www.scielo.org.mx/scielo.php?script=sci_arttext\&pid =S0568-25172007000200001

Taiz, L. y Zeiger, E. (1998). Plant physiology (2a ed.). Sunderland, Massachusetts, EE. UU.: Sinauer Associates, Inc.

Thanos, C. y Georghiou, K. (1988). Osmoconditioning enhances cucumber and tomato seed germinability under adverse light conditions. Israel Journal of Botany, 37, 1-10. Recuperado de: http://users.uoa. gr/ kgeorghi/files/10_osmocond_t_conditions.pdf

Trigo, M., Nedel, J., Garcia, A. y Trigo, L. (1999). Efeitos do condicionamento osmótico com soluções aeradas de nitrato de potássio no desempenho de sementes de cebola. Revista Brasileira de Sementes, 21, 139144. 